\title{
On a Model for the Growth of an Invasive Avascular Tumor
}

\author{
J. A. J. Avila ${ }^{1, *}$ and G. Lozada-Cruz, ${ }^{2}$ \\ ${ }^{1}$ Department of Mathematics and Statistics, Universidade Federal de São João del Rei, 36307-904, São João del Rei, MG, Brazil \\ ${ }^{2}$ Department of Mathematics,IBILCE, Universidade Estadual Paulista, 15054-000, São José do Rio Preto, São Paulo, Brazil
}

Received: 17 Jan. 2013, Revised: 20 May. 2013, Accepted: 21 May. 2013

Published online: 1 Sep. 2013

\begin{abstract}
This paper is devoted to study the 1D model of invasive avascular tumor growth, which takes into account cell division, death, and motility, proposed by Kolobov and collaborators in 2009. First, we examine the existence and uniqueness of the solution to this model. Second, we studied qualitatively and numerically the traveling wave solutions. Finally, we show some numerical simulations for the cell density and nutrient concentration.
\end{abstract}

Keywords: Invasive avascular tumor, traveling wave, existence of solution, numerical simulation.

\section{Introduction}

Cancer is a leading cause of death worldwide and accounted for 7.6 million deaths (around 13\% of all deaths) in 2008, [1]. On the other hand, in Brazil, the National Cancer Institute (NCI) released estimates of cancer incidence for 2012, which will be valid also for 2013. They point to the occurrence of approximately 518,510 new cases of cancer, [2]. The tumors principals in males are due to non-melanoma skin cancer and prostate cancer. For females stand out from the non-melanoma skin cancer and breast cancer.

It is considered a tumor as a colony of cancer cells (live and dead) that grow uncontrollably, surrounded by normal tissue. Among the various types of tumors, the most common are: solid tumors and invasive tumors, the difference is in the consistency of the expansive power. Solid tumors grow expanding as a compact mass with well-defined edge between cancer cells and normal tissue, however, the invasive tumors grow rapidly expanding but the fraction of cancer cells in the tissue is low.

The tumors are generally vascular, i.e, there are a network of blood vessels in it, with great possibilities to develop angiogenesis. Initially these tumors can be considered without the presence of blood vessels, which are called avascular tumors. Thus, the concentration of nutrients (oxygen) diffuses to tumor cells from remote enough blood vessels. In Figure 1 we showed a schematic representation of an avascular tumor with necrotic region in the center. The mathematical tool that models the

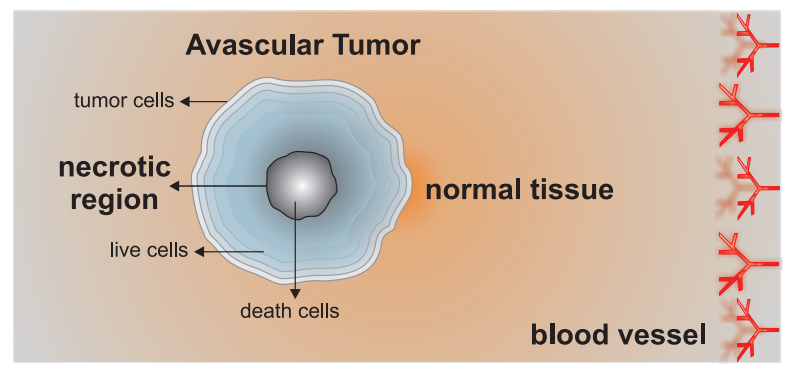

Fig. 1: Schematic illustration showing the 2D avascular tumor.

growth of tumors are the partial differential equations of parabolic type. In the case of invasive avascular tumors are considered cell division, death and motility, as essential variables in the dynamics of cell density and nutrient concentration governed by reaction-diffusion equation.

The modeling of the avascular tumor growth is the first step towards building fully vascularized tumor models. Some references regarding the qualitative analysis of the dynamics of solid avascular tumor growth are found in [3,4]. Sherratt and Chaplain [5] formulate a new mathematical model for avascular tumor growth. Ferreira et al. [6] use the equation of reaction-diffusion model for the growth of a tumor avascular with numerical

\footnotetext{
*Corresponding author e-mail: avila_jaj@ufsj.edu.br
} 
results. Jiang et al. [7] provide a multiscale model (levels: cellular, subcellular and extracellular) for growth of avascular tumors with numerical results. Roose et al [8] show an review outline of a number of illustrative mathematical models describing the growth of avascular tumors. Bresch et al. [9] reported a viscoelastic model for the growth of a avascular tumor that describes the evolution of three components: sane tissue, cancer cells and extracellular medium. Kolobov et al. [10] study the autowaves in a model of invasive avascular tumor growth with 1-d numerical results. Again, Bresch et al. [11] studied the growth an solid avascular tumor in two and three dimensions with a focus on numerical methods.

This work continues the studies of Kolobov et al. [10] adding the part of mathematical analysis: existence and uniqueness of the solution, and continues dependence of the initial data. Finally, we present other numerical results, also.

The organization of this work is as follows. In Section 2 we present the mathematical model for invase avascular tumor growth. Section 3 is devoted to show the problem (1) - (2) is well posed in a suitable function space and, in particular we study the traveling waves. Finally, in Section 4 , we present some numerical simulations for the problem (1) - (2).

\section{The model}

According Kolobov et al. in [10] the system of nonlinear partial differential equations that models the growth of an one-dimensional invasive avascular tumor is given by the reaction-diffusion equation

$$
\begin{cases}a_{t}=D_{a} a_{x x}-P(s) a+B a, & -\infty<x<+\infty, t \geqslant 0 \\ s_{t}=D_{s} s_{x x}-q a, & -\infty<x<+\infty, t \geqslant 0\end{cases}
$$

subject with the boundary conditions

$$
\begin{gathered}
\left(a, s_{x}\right) \rightarrow(0,0) \text { when } x \rightarrow-\infty \\
(a, s) \rightarrow(0,1) \text { when } x \rightarrow+\infty,
\end{gathered}
$$

where

$$
\begin{aligned}
& a=a(x, t): \text { cell density (density of live tumor cells) } \\
& s=s(x, t): \text { nutrient concentration }
\end{aligned}
$$

and the parameters are defined by

$$
\begin{aligned}
D_{a} & : \text { diffusion coefficient of tumor cells } \\
D_{s} & : \text { diffusion coefficient of oxygen } \\
B & : \text { division rate of live cells } \\
P_{m} & : \text { maximal death rate } \\
s_{\text {crit }} & : \text { critical nutrient concentration } \\
\varepsilon & : \text { characteristic deviation of } s \text { from } s_{\text {crit }} \\
q & : \text { nutrient consumption rate of live tumor cells } \\
P(s) & : \text { cell death rate. } P(s)=\frac{P_{m}}{2}\left[1-\tanh \left(\frac{s-s_{\text {crit }}}{\varepsilon}\right)\right] .
\end{aligned}
$$

This model was obtained with the following assumptions:

1.The surroundings of the tumor, corresponding to normal tissue, do not prevent the movement of live tumor cells and proliferation.

2.The growth of the tumor, in normal tissue, is without the development of capillary networks.

3.Although cell division requires a large variety of nutrients, this model assumes that the oxygen is the only nutrient and, the missed him, causes the death of live tumor cells.

4.The diffusion of oxygen begins in blood vessels distant from the tumor, as shown in Figure 1.

\section{Mathematical analysis}

In this section we study the existence and uniqueness of the solution, and continues dependence of the initial data. Also, we analyze qualitatively the solutions traveling wave type.

\subsection{Well-posed problem}

We consider the system (1) with initial conditions

$$
a(x, 0)=a_{0}(x) \geqslant 0, s(x, 0)=s_{0}(x) \geqslant 0, \forall x \in \mathbb{R} .
$$

For $1 \leqslant p<\infty$ we define the Banach space $X_{p}$ by $X_{p}=\{u: \mathbb{R} \rightarrow \mathbb{R} ; u$ is bounded, uniform continuous and

$$
\left.u \in L^{p}(\mathbb{R})\right\}
$$

endowed with the norm

$$
\|u\|_{p}=\sup _{\tau \in \mathbb{R}}|u(\tau)|+\left(\int_{-\infty}^{+\infty}|u(\tau)|^{p} d \tau\right)^{1 / p} .
$$

For $p \geqslant q$ we have the continuous inclusion $W^{1, q}(\mathbb{R}) \subset$ $X_{p}$. Now, let $n \in \mathbb{N}$ and denote by $X_{p}^{(n)}$ the Banach space $X_{p}^{(n)}=\left\{u \in X_{p}: u^{\prime}, u^{\prime \prime}, \cdots, u^{(n)} \in L^{p}(\mathbb{R})\right\}$ with the norm

$$
\|u\|_{p}^{(n)}=\sum_{j=0}^{n}\left\|u^{(j)}\right\|_{p}, u \in X_{p}^{(n)} .
$$

The equation (1) joint with (3) can be rewrite in the space $X=X_{p} \times X_{p}$ as an abstract evolution equations

$$
\left\{\begin{aligned}
u_{t} & =A u+F(u) \\
u(0) & =u_{0}
\end{aligned}\right.
$$

where $F: X \rightarrow X$ is a nonlinear function given by $F(u)=$ $(-P(s) a,-q a), u=(a, s), u_{0}=\left(a_{0}, s_{0}\right)$ and $A: D(A) \subset$ $X \rightarrow X$ is given by

$$
\begin{gathered}
D(A)=\left\{(a, s) \in X: a, s \in X_{p}^{(2)},\left(a, s_{x}\right) \rightarrow(0,0), x \rightarrow-\infty,\right. \\
(a, s) \rightarrow(0,1), x \rightarrow+\infty\} \\
A u=\left(D_{a} a_{x x}+B a, D_{s} s_{x x}\right) .
\end{gathered}
$$


Using variation of constants formula, the solution of (4) is given by

$$
u(t)=e^{t A} u_{0}+\int_{0}^{t} e^{(t-\tau) A} F(u(\tau)) d \tau, t \geqslant 0
$$

where

$$
\begin{aligned}
e^{t A} & =\left(\begin{array}{cc}
T_{1}(t) & 0 \\
0 & T_{2}(t)
\end{array}\right) \\
\left(T_{1}(t) a_{0}\right)(x) & =e^{B t} \int_{\mathbb{R}} \Gamma_{1}(t, x-y) a_{0}(y) d y \\
\left(T_{2}(t) s_{0}\right)(x) & =\int_{\mathbb{R}} \Gamma_{2}(t, x-y) s_{0}(y) d y .
\end{aligned}
$$

The functions $\Gamma_{1}$ and $\Gamma_{2}$ are the heat kernel associated with the parabolic equations $a_{t}=D_{a} a_{x x}$ and $s_{t}=D_{s} s_{x x}$ respectively.

Note that the equations $a_{t}=D_{a} a_{x x}+B a$ and $s_{t}=D_{s} s_{x x}$ generate the linear semigroups $T_{1}(t)$ and $T_{2}(t)$, respectively.

Thus, the equation (5), for $t \geqslant 0$, take the form

$$
\begin{aligned}
& a\left(\cdot, t, u_{0}\right)=T_{1}(t) a_{0}-\int_{0}^{t} T_{1}(t-\tau) P(s(\tau)) a(\tau) d \tau, \\
& s\left(\cdot, t, u_{0}\right)=T_{2}(t) s_{0}-q \int_{0}^{t} T_{2}(t-\tau) a(\tau) d \tau
\end{aligned}
$$

Definition 1. We say that the function $u:[0,+\infty) \rightarrow X$ is a mild solution of (4) if $u \in C([0, \infty), X)$ and satisfies (5).

We have the following result on existence and uniqueness of (1)-(2).

Theorem 1.Assume that $F$ is global Lipschitz continuous. For any $u_{0}=\left(a_{0}, s_{0}\right) \in X$ the system (1) with the boundary conditions (2) has a unique mild solution $u\left(x, t, u_{0}\right)=\left(a\left(x, t, u_{0}\right), s\left(x, t, u_{0}\right)\right)$ for all $t \geqslant 0$ with $u\left(\cdot, 0, u_{0}\right)=u_{0}$.

Proof. From the definition of $P$ and $F(u)=(-P(s) a,-q a)$, it is not difficult to see that there is $L>0$ such that $\|F(u)-F(v)\| \leqslant L\|u-v\|$ for all $u, v \in X$. Thus, as a consequence of the abstract results of [12], there exists a unique mild solution for (1). $X$ by

Now, define the family of operators $\{T(t): t \geqslant 0\}$ on

$$
\begin{aligned}
(T(t) \phi)(x) & =e^{t A} \phi(x) \\
& =u(x, t, \phi) \\
& =(a(x, t, \phi), s(x, t, \phi)), \forall x \in \mathbb{R}, t \geqslant 0 .
\end{aligned}
$$

For any $\left(t_{0}, \phi_{0}\right) \in[0, \infty) \times X$ we have

$$
\begin{array}{r}
\left\|T(t)(\phi)-T\left(t_{0}\right) \phi_{0}\right\| \leqslant \\
\left\|T(t) \phi-T(t) \phi_{0}\right\|+ \\
\left\|T(t) \phi_{0}-T\left(t_{0}\right) \phi_{0}\right\|
\end{array}
$$

From the inequality (8), follows that $T(t) \phi$ is continuous at $\left(t_{0}, \phi_{0}\right)$, and thus the solution $u(x, t, \phi)$ of (1) depends continuously on respect to initial data.

From the definition of $\{T(t): t \geqslant 0\}$ it follows immediately that

i) $T(0) u_{0}=u\left(\cdot, 0, u_{0}\right)=u_{0}$.

ii) $T\left(t_{1}+t_{2}\right) u_{0}=T\left(t_{1}\right) T\left(t_{2}\right) u_{0}, \forall t_{1}, t_{2} \geqslant 0$, this property is a consequence of uniqueness of solution of (1).

iii) The map $[0, \infty) \times X \ni\left(t, u_{0}\right) \mapsto T\left(t, u_{0}\right)=u\left(t, u_{0}\right) \in X$ is continuous, this follows of inequality (8).

\subsection{Traveling waves}

A travelling wave of (1), (2) is a solution of the type $(a(x, t), s(x, t))=(A(\xi), S(\xi)) \in C^{2}(\mathbb{R}) \times C^{2}(\mathbb{R})$ where $\xi=x-c t$, the functions $A$ and $S$ are the profile of the travelling wave and $c \in \mathbb{R}$ is the speed of propagation of the wave. The existence of this kind of solutions can be found in [12-14] among others.

The functions $A$ and $S$ reduce system (1) in the following systems of ODEs of second order

$$
\left\{\begin{array}{r}
D_{a} A^{\prime \prime}(\xi)+c A^{\prime}(\xi)-P(S) A(\xi)+B A(\xi)=0 \\
D_{s} S^{\prime \prime}(\xi)+c S^{\prime}(\xi)-q A(\xi)=0
\end{array}\right.
$$

where $-\infty<\xi<+\infty$, with the boundary conditions

$$
\left\{\begin{array}{l}
(A, S)(\xi) \rightarrow(0, \sigma) \text { as } \xi \rightarrow-\infty \\
(A, S)(\xi) \rightarrow(0,1) \text { as } \xi \rightarrow+\infty
\end{array}\right.
$$

where $\sigma$ is a constant corresponding to the limit oxygen concentration at $\xi \rightarrow-\infty$.

Note that the points $(0, \sigma)$ and $(0,1)$ are stationary points of (9). Now, linearizing the system (9) around the stationary point $(0, \sigma)$ we have

$$
\left\{\begin{array}{r}
D_{a} A_{-}^{\prime \prime}(\xi)+c A_{-}^{\prime}(\xi)+(B-P(\sigma)) A_{-}(\xi)=0 \\
D_{s} S_{-}^{\prime \prime}(\xi)+c S_{-}^{\prime}(\xi)-q A_{-}(\xi)=0
\end{array}\right.
$$

where $-\infty<\xi<+\infty$. Also, linearizing the system (9) around the stationary point $(0,1)$ we have

$$
\left\{\begin{array}{r}
D_{a} A_{+}^{\prime \prime}(\xi)+c A_{+}^{\prime}(\xi)+B A_{+}(\xi)=0 \\
D_{s} S_{+}^{\prime \prime}(\xi)+c S_{+}^{\prime}(\xi)-q A_{+}(\xi)=0
\end{array}\right.
$$

where $-\infty<\xi<+\infty$.

Introducing a vector with the components $x_{1}=A_{-}$, $x_{2}=A_{-}^{\prime}, x_{3}=S_{-}, x_{4}=S_{-}^{\prime}$, the system (11) can be written as a system of ODEs of first order

$$
\dot{\mathbf{x}}=\Lambda \mathbf{x}
$$


where

$$
\begin{aligned}
\Lambda & =\left(\begin{array}{cccc}
0 & 1 & 0 & 0 \\
\frac{P(\sigma)-B}{D_{a}} & -\frac{c}{D_{a}} & 0 & 0 \\
0 & 0 & 0 & 1 \\
\frac{q}{D_{s}} & 0 & 0 & -\frac{c}{D_{s}}
\end{array}\right), \\
\mathbf{x} & =\left(x_{1}, x_{2}, x_{3}, x_{4}\right)^{\mathrm{T}} .
\end{aligned}
$$

Similarly, putting $y_{1}=A_{+}, y_{2}=A_{+}^{\prime}, y_{3}=S_{+}, y_{4}=S_{+}^{\prime}$, the system (12) can be written as a system of ODEs of first order

$$
\dot{\mathbf{y}}=\Xi \mathbf{y}
$$

where

$$
\begin{aligned}
& \Xi=\left(\begin{array}{cccc}
0 & 1 & 0 & 0 \\
-\frac{B}{D_{a}} & -\frac{c}{D_{a}} & 0 & 0 \\
0 & 0 & 0 & 1 \\
\frac{q}{D_{s}} & 0 & 0 & -\frac{c}{D_{s}}
\end{array}\right), \\
& \mathbf{y}=\left(y_{1}, y_{2}, y_{3}, y_{4}\right)^{\mathrm{T}} .
\end{aligned}
$$

The matrices $\Lambda$ and $\Xi$ have eigenvalues respectively

$$
\begin{aligned}
& \mu_{1}^{-}=0, \mu_{2}^{-}=-\frac{c}{D_{s}}, \mu_{3,4}^{-}=\frac{-c \pm \sqrt{\Delta^{-}}}{2 D_{a}} \\
& \mu_{1}^{+}=\mu_{1}^{-}, \mu_{2}^{+}=\mu_{2}^{-}, \mu_{3,4}^{+}=\frac{-c \pm \sqrt{\Delta^{+}}}{2 D_{a}}
\end{aligned}
$$

where

$$
\begin{aligned}
& \Delta^{-}=c^{2}+4(P(\sigma)-B) D_{a} \\
& \Delta^{+}=c^{2}-4 B D_{a}
\end{aligned}
$$

and the corresponding eigenvectors are given, respectively, by

$$
\begin{aligned}
& \mathbf{k}_{1}^{-}=(0,0,1,0)^{\mathrm{T}}, \quad \mathbf{k}_{2}^{-}=\left(0,0,-\frac{D_{s}}{c}, 1\right)^{\mathrm{T}} \\
& \mathbf{k}_{3}^{-}=\left(\begin{array}{c}
-\frac{c\left(D_{s}-2 D_{a}\right)+D_{s} \sqrt{\Delta^{-}}}{2 q D_{a}} \\
\frac{D_{a}\left[c\left(c-\sqrt{\Delta^{-}}\right)+2(P(\sigma)-B) D_{s}\right]}{2 q D_{a}^{2}} \\
-\frac{2 D_{a}}{c+\sqrt{\Delta^{-}}} \\
1
\end{array}\right) \\
& \mathbf{k}_{4}^{-}=\left(\begin{array}{c}
-\frac{c\left(D_{s}-2 D_{a}\right)-D_{s} \sqrt{\Delta^{-}}}{2 q D_{a}} \\
\frac{\left(-c+\sqrt{\Delta^{-}}\right)\left[-c\left(D_{s}-2 D_{a}\right)+D_{s} \sqrt{\Delta^{-}}\right]}{4 q D_{a}^{2}} \\
-\frac{2 D_{a}}{c+\sqrt{\Delta^{-}}} \\
1
\end{array}\right)
\end{aligned}
$$

$$
\mathbf{k}_{3}^{+}=\left(\begin{array}{c}
\mathbf{k}_{1}^{+}=\mathbf{k}_{1}^{-}, \quad \mathbf{k}_{2}^{+}=\mathbf{k}_{2}^{-} \\
-\frac{c\left(D_{s}-2 D_{a}\right)+D_{s} \sqrt{\Delta^{+}}}{2 q D_{a}} \\
\frac{c\left(D_{s}-D_{a}\right)\left(c+\sqrt{\Delta^{+}}\right)-2 B D_{a} D_{s}}{2 q D_{a}^{2}} \\
-\frac{2 D_{a}}{c+\sqrt{\Delta^{+}}} \\
1 \\
\mathbf{k}_{4}^{+}=\left(\begin{array}{c}
\frac{c\left(D_{s}-2 D_{a}\right)-D_{s} \sqrt{\Delta^{+}}}{2 q D_{a}} \\
\frac{c\left(D_{s}-D_{a}\right)\left(c-\sqrt{\Delta^{+}}\right)-2 B D_{a} D_{s}}{2 q D_{a}^{2}} \\
\frac{2 D_{a}}{c+\sqrt{\Delta^{+}}} \\
1
\end{array}\right)
\end{array}\right.
$$

Thus, for the system (13), we have the following linear independent solutions

$$
\mathbf{x}_{i}(\xi)=\mathbf{k}_{i}^{-} e^{\mu_{i}^{-} \xi}, \quad i=1, \cdots, 4
$$

From (20) we have $\lim _{\xi \rightarrow-\infty} \mathbf{x}_{1}(\xi)=\mathbf{k}_{1}^{-}, \mathbf{x}_{2}(\xi)=\mathbf{k}_{2}^{-} e^{\mu_{2}^{-} \xi}$ and $\mathbf{x}_{4}(\xi)=\mathbf{k}_{4}^{-} e^{\mu_{4}^{-} \xi}$ are unbounded solutions when $\xi \rightarrow-\infty$ if $c>0$ and $P(\sigma)>B$ respectively. And, $\mathbf{x}_{3}(\xi)=\mathbf{k}_{3}^{-} e^{\mu_{3}^{-} \xi}$ is bounded when $\xi \rightarrow-\infty$.

If $\mu_{3}^{-}$is a real positive (negative) number the stationary point $(0, \sigma)$ of (11) is saddle (stable) node and if $\mu_{3}^{-}$is a complex number the stationary point $(0, \sigma)$ of $(11)$ is a stable focus.

And for the system (15), we have the following linear independent solutions

$$
\mathbf{y}_{i}(\xi)=\mathbf{k}_{i}^{+} e^{\mu_{i}^{+} \xi}, \quad i=1, \cdots, 4 .
$$

From (21) we have $\lim _{\xi \rightarrow+\infty} \mathbf{y}_{1}(\xi)=\mathbf{k}_{1}^{+}, \lim _{\xi \rightarrow+\infty} \mathbf{y}_{2}(\xi)=\mathbf{0}$ and the other solutions $\mathbf{y}_{i}(\xi)=\mathbf{k}_{i}^{+} e^{\mu_{i}^{+} \xi}, i=2,3$ also remains bounded when $\xi \rightarrow+\infty$ if $c \geqslant 2 \sqrt{B D_{a}}$. Thus, we have that the stationary point $(0,1)$ of $(12)$ is either a stable node or a stable focus.

\section{Numerical results}

In this section we will find numerically by finite difference method, a solution of type traveling wave, and full solution of our problem (1) - (2). 


\subsection{Dimensionless equations}

The units of measurements for the cell density and nutrients concentration is, respectively,

$$
c\left[\text { cell } / \mathrm{cm}^{3}\right] \text { and } s\left[\mathrm{~mol} / \mathrm{cm}^{3}\right] .
$$

The values and units of measurement of the parameters are taken from Kolobov et al. [10],

$$
\begin{array}{rlrl}
D_{a} & =2.5 \times 10^{-8}\left[\mathrm{~cm}^{2} / \mathrm{s}\right], & D_{s} & =2.5 \times 10^{-5}\left[\mathrm{~cm}^{2} / \mathrm{s}\right] \\
B & =10^{-7}[1 / \mathrm{s}], & P_{m} & =0.2 \times 10^{-6}[1 / \mathrm{s}] \\
s_{\text {crit }} & =0.3 \times 10^{-7}\left[\mathrm{~mol} / \mathrm{cm}^{3}\right], & \varepsilon & =10^{-9}\left[\mathrm{~cm}^{3} / \mathrm{mol}\right] \\
q=10^{-20}[\mathrm{~mol} / \mathrm{s} \cdot \text { cell }] . &
\end{array}
$$

The characteristic scales are:

$$
\begin{gathered}
\text { Length scale }: L_{0}=5 \times 10^{-2}[\mathrm{~cm}] \\
\text { Time scale }: T_{0}=10^{6}[\mathrm{~s}] \\
\text { Density scale }: a_{\max }=10^{7}\left[\mathrm{cell} / \mathrm{cm}^{3}\right] \\
\text { Concentration scale }: s_{\max }=10^{-7}\left[\mathrm{~mol} / \mathrm{cm}^{3}\right] .
\end{gathered}
$$

The independent and dependent dimensionless variables are given by

$$
x^{*}=x / L_{0}, t^{*}=t / T_{0}, a^{*}=a / a_{\max }, s^{*}=s / s_{\max }
$$

We have used the following dimensionless data:

$$
\begin{gathered}
D_{a}^{*}=10, \quad D_{s}^{*}=10^{4}, \quad B^{*}=10^{-1}, P_{m}^{*}=0.2, s_{\text {crit }}^{*}=0.3, \\
\varepsilon^{*}=10^{-2}, \quad q^{*}=1, \quad c_{\min }=2 \sqrt{2}, c=c_{\min }, \quad \sigma=0.18
\end{gathered}
$$

Henceforth, the equations are written in dimensionless form.

\subsection{Traveling waves}

Rewriting the equation (9) in the following form

$$
\left\{\begin{array}{l}
A^{\prime \prime}=p_{1} A^{\prime}+q_{1}(S) A \\
S^{\prime \prime}=p_{2} S^{\prime}+q_{2} A
\end{array}\right.
$$

where

$$
\begin{aligned}
& p_{1}=-\frac{c}{D_{a}}, \quad q_{1}(S)=\frac{1}{D_{a}}(P(S)-B), \\
& p_{2}=-\frac{c}{D_{s}}, \quad q_{2}=\frac{q}{D_{s}}
\end{aligned}
$$

joint with the boundary conditions

$$
\begin{aligned}
& A(-L)=0=A(L) \\
& S(-L)=\sigma, \quad S(L)=1,
\end{aligned}
$$

where $\xi \in(-L, L)$ and $L>0$ is large.

Taking $L=1000$ and using finite difference method we have a numerical solutions for cell density and nutrient concentration which are showed in the Figure 2 and 3 , respectively.

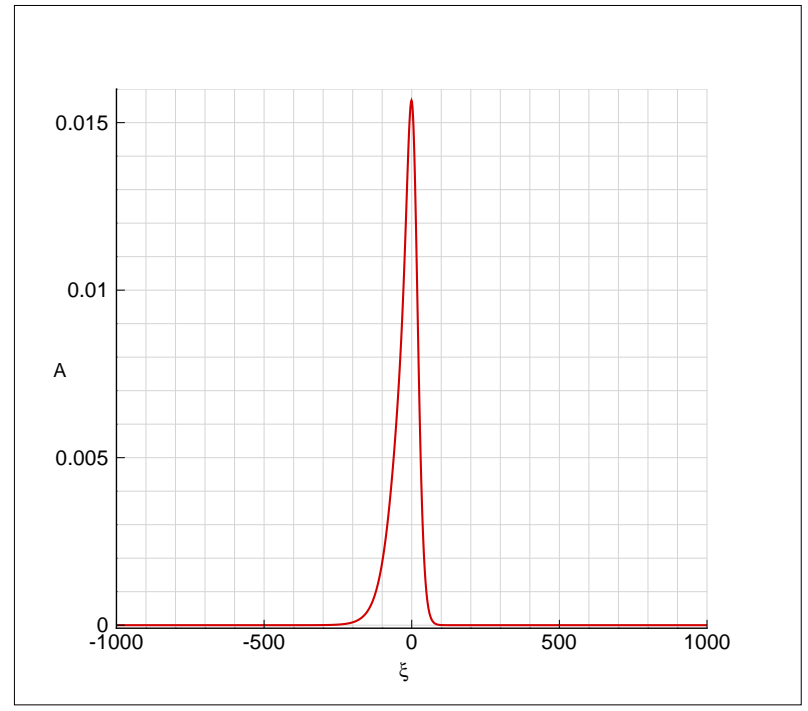

Fig. 2: Cell density profile $A(\xi)$.

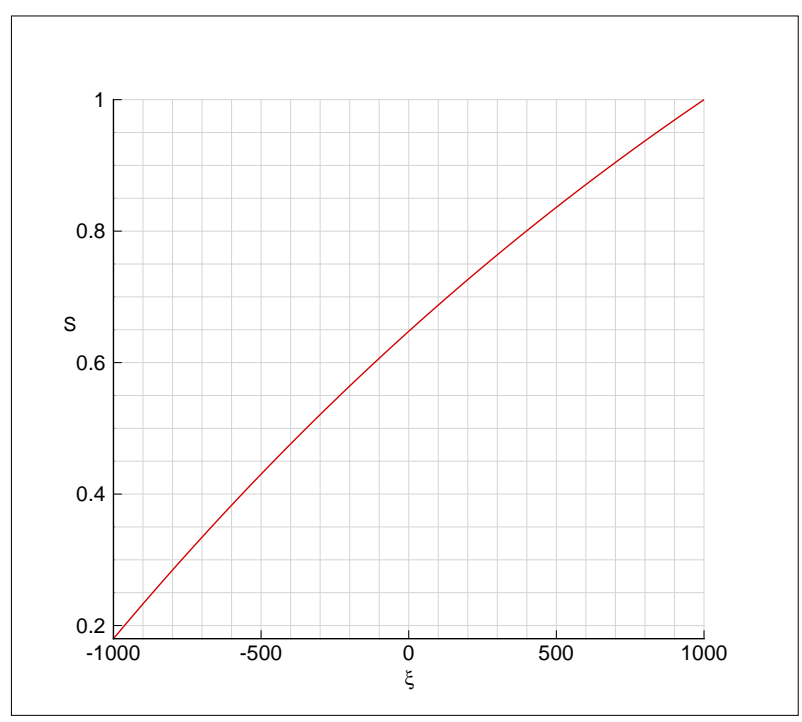

Fig. 3: Nutrient concentration profile $S(\xi)$.

\subsection{Numerical solution}

For the numerical solution of problem (1) - (2) we used the classic and unconditionally stable Crank-Nicolson Method. The domain $(-L, L)$ is discretized with uniform grid of $N=1200$ elements and $L=2000$. The sizes of spatial and temporal steps are, respectively, $h=2 L / N$ and $k=v_{0} h / c$, where $v_{0}=0.26$. The initial condition is given by gaussian function $a(x, 0)=0.1 e^{-0.0025 x^{2}}$ and constant function $s(x, 0)=1$.

The asymptotic behavior of live cell density, at each point where it reaches its maximum value, is showed in 
the Figure 4. Note that of stationary state is attained in $t \approx 200$, i.e, in approximately 6.5 years. In Figure 5 and 6

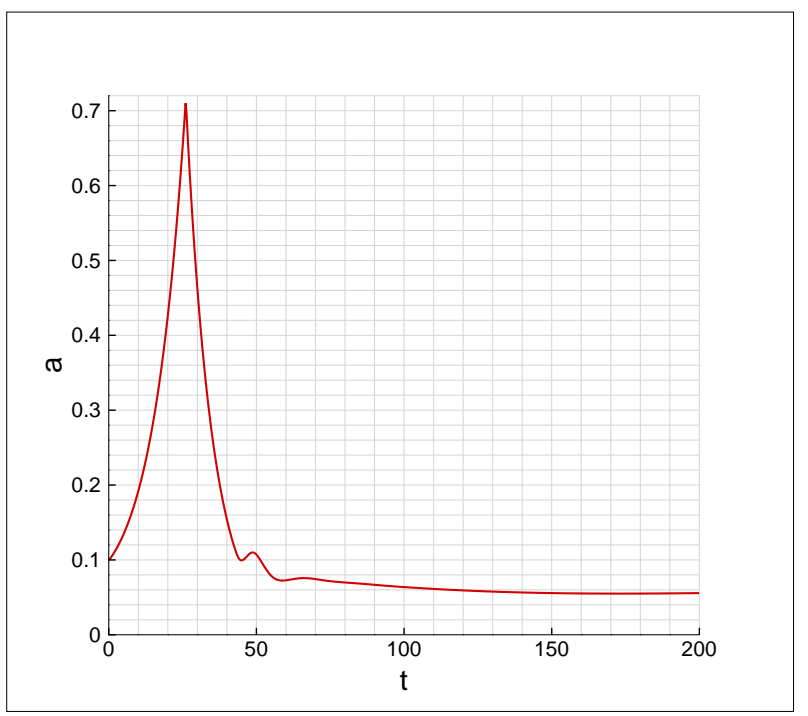

Fig. 4: Asymptotic behavior of maximal live cell density $a$.

we showed, in $3 \mathrm{D}$, the density profile of live tumor cells and nutrient concentration profile, respectively, the longtime. We note that at steady state, the density profile of live tumor cells approaches the solution of type traveling wave, given, in the Figure 2.

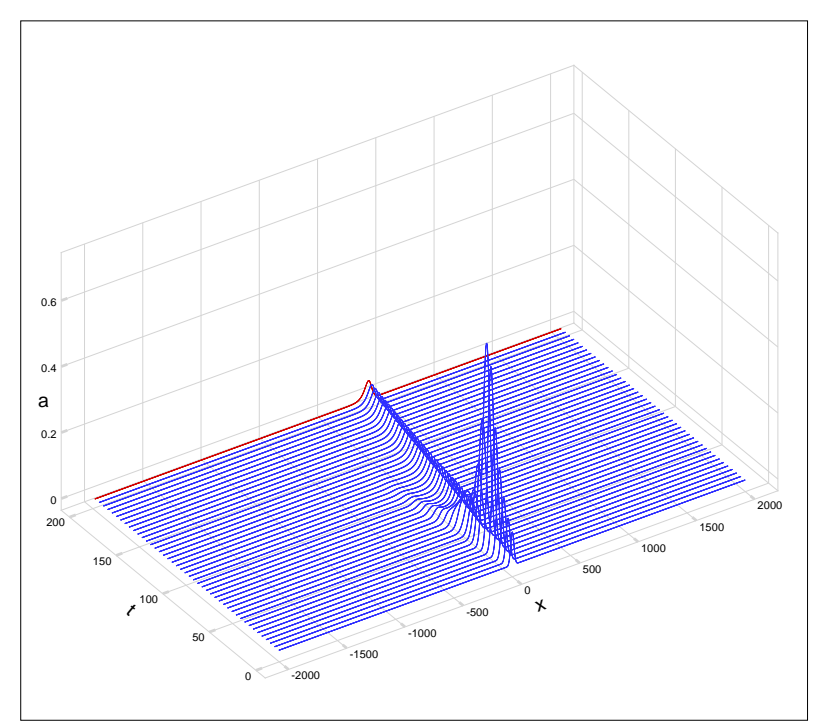

Fig. 5: The profile of live cell density the long-time.

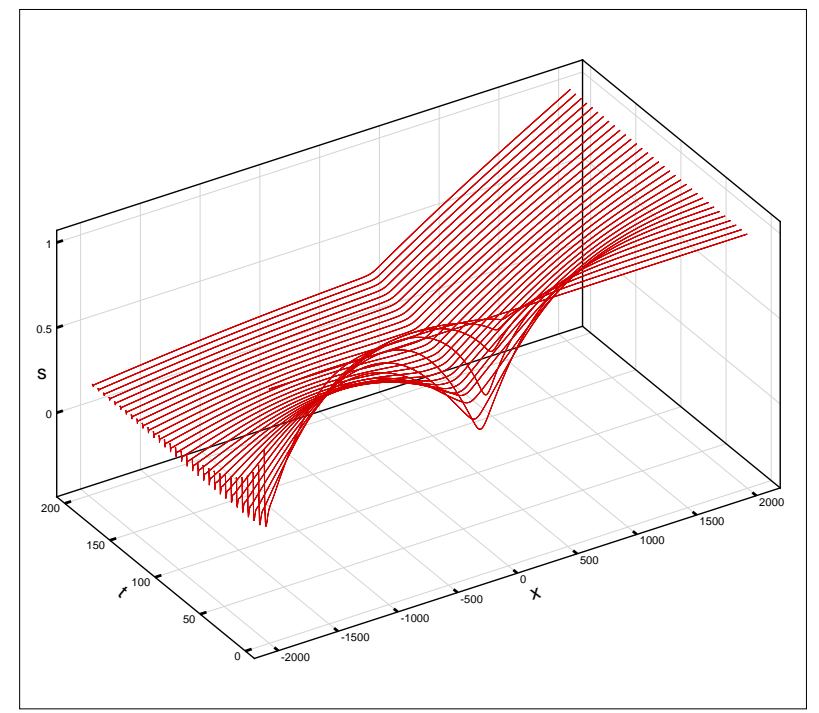

Fig. 6: The profile of nutrient concentration density for long-time

\section{Conclusion}

The problem of growth of an invasive avascular tumor, one dimensional, is well-posed. Physically, we observe in figures and in numerical simulations that the tumor grows in the direction where there are nutrients (oxygen). It is observed that it is invasive and aggressive from the beginning to about $t=60$ ( 2 years), immediately after a relaxation which is going to reach steady state, at approximately $t=200$ (6.5 years), where the density of live tumoral cells is very low. The tumor grows because the tumor cells die. Thus, the volume of necrotic region increases.

\section{References}

[1] World Healt Organization: Media centre. Cancer, Fact sheet No297, Reviewed January, (2013).

[2] Estimativa 2012: Incidência de Câncer no Brasil. Instituto Nacional de Câncer José Alencar Gomes da Silva, Coordenação Geral de Ações Estratégicas, Coordenação de Prevenção e Vigilância. Rio de Janeiro: Inca, 118 (2011).

[3] Ward, J. P. King, J. R., Mathematical modelling of avasculartumour growth. IMA Journal of Mathematics Applied in Medicine \& Biology, 14, 39-69 (1997).

[4] Byrne, Helen, M. A weakly nonlinear analysis of a model of avascular solid tumour growth. Journal Mathematical Biology, 39, 59-89 (1999).

[5] Sherratt, Jonathan A.; Chaplain, Mark A. J., A new mathematical model for avascular tumour growth. Mathematical Biology, 43, 291-312 (2001).

[6] Ferreira Jr., S. C.; Martins, M. L.; Vilela, M. J., Reactiondiffusion model for the growth of avascular tumor. Physical Review E, 65, 021907, (2002). 
[7] Jiang, Yi; Pjesivac-Grbovic, J.; Cantrell, Charles; Freyer, J. P., A Multiscale model for avascular tumor growth. Biophysical Journal, 89, 3884-3894 (2005).

[8] Roose, T.; Chapman, S. J.; Maini, P. K., Mathematical Models of Avascular Tumor Growth. SIAM Review, 49, 179208 (2007).

[9] Bresch, Didier; Colin, Thierry; Grenier, Emmanuel; Ribba, Benjamin; Saut, Oliver, A viscoelastic model for avascular tumor growth. Discrete and Continuous Dynamical Systems, supplement, 101-108 (2009).

[10] Kolobov, A. V., Gubernov, V. V. and Polezhaev, A. A., Autowaves in a model of invasive tumor growth. Biophysics, 54, 232-237 (2009).

[11] Bresch, Didier; Colin, Thierry; Grenier, Emmanuel; Ribba, Benjamin; Saut, Oliver, Computational modeling of solid tumor growth: The avascular stage. SIAM J. Sci. Comput, 32, 2321-2344 (2010).

[12] Henry, D., Geometric theory of semilinear parabolic equations, Lect. Notes in Math. Springer, 840, (1981).

[13] Aronson, D. G.; Weinberger, H. F., Multidimensional nonlinear diffusion arising in population genetics. Adv. in Math., 30, 33-76 (1978).

[14] Volpert, Aizik I.; Volpert, Vitaly A.; Volpert, Vladimir A., Traveling wave solutions of parabolic systems. Translations of Mathematical Monographs. American Mathematical Society, Providence, RI, 140, (1994).

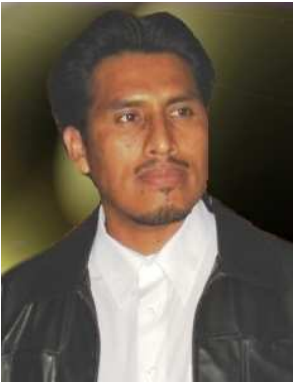

Jorge Andrés Julca Avila, $\mathrm{PhD}$ in Mechanical Engineering from USP - University of São Paulo. Assistant Professor at the Department of Mathematics of UFSJ - Federal University of São João del-Rei. Has experience in Applied Mathematics emphasizing Numerical Analysis, Functional Analysis and PDEs. For the moment, the major interests are in the Navier-Stokes Equations, Non Newtonian Fluids and Finite Elements. Is also experienced in Mechanical Engineering with emphasis in Fluid Mechanics and Fluid Dynamics. Is member of the Scientific Committee of UFSJ.

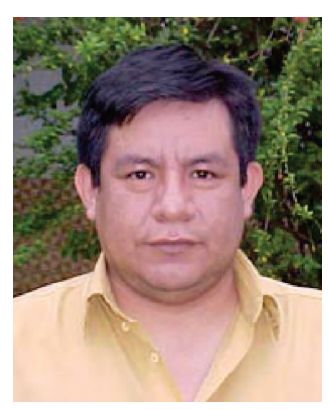

Germán Jesús Lozada Cruz, PhD in Mathematics (2001) from University of São Paulo at São Carlos. Assistant professor at the Department of Mathematics of State University of São Paulo. Has experience in Mathematics with emphasis in Partial Differential Equations.

The areas of interest are asymptotic behavior of reaction diffusion equations in dumbbell domains, existence of attractors and continuity of attractors with relation to small parameter. 